\title{
Maps of open chromatin highlight cell type-restricted patterns of regulatory sequence variation at hematological trait loci
}

\author{
Dirk S. Paul, ${ }^{1,2,13,16}$ Cornelis A. Albers, ${ }^{1,3,4,13}$ Augusto Rendon, ${ }^{3,5,6,13}$ Katrin Voss, ${ }^{3}$ \\ Jonathan Stephens, ${ }^{3}$ HaemGen Consortium, ${ }^{15}$ Pim van der Harst, ${ }^{7,8}$ \\ John C. Chambers, ${ }^{9,10,11,12}$ Nicole Soranzo, ${ }^{1}$ Willem H. Ouwehand, ${ }^{1,3,14}$ \\ and Panos Deloukas ${ }^{1,14,16}$
}

\begin{abstract}
${ }^{1}$ Wellcome Trust Sanger Institute, Hinxton, Cambridge CB10 1SA, United Kingdom; ${ }^{2}$ UCL Cancer Institute, University College London, London WC1E 6BT, United Kingdom; ${ }^{3}$ Department of Haematology, University of Cambridge and National Health Service (NHS) Blood and Transplant, Cambridge CB2 OPT, United Kingdom; ${ }^{4}$ Department of Human Genetics, Radboud University Nijmegen Medical Center, 6500 HB Nijmegen, The Netherlands; ${ }^{5}$ MRC Biostatistics Unit, Institute of Public Health, Cambridge CB2 0SR, United Kingdom; ${ }^{6}$ NIHR Biomedical Research Centre, Cambridge CB2 OPT, United Kingdom; ${ }^{7}$ Department of Cardiology, ${ }^{8}$ Department of Genetics, University of Groningen, University Medical Center Groningen, 9713 GZ Groningen, The Netherlands; ${ }^{9}$ Department of Epidemiology and Biostatistics, Imperial College London, London W2 1NY, United Kingdom; ${ }^{10}$ Imperial College Healthcare NHS Trust, Hammersmith Hospital, London W12 OHS, United Kingdom; ${ }^{11}$ Royal Brompton and Harefield Hospitals NHS Trust, London SW3 6NP, United Kingdom; ${ }^{12}$ Ealing Hospital NHS Trust, Southall, Middlesex UB1 3HW, United Kingdom
\end{abstract}

\begin{abstract}
Nearly three-quarters of the 143 genetic signals associated with platelet and erythrocyte phenotypes identified by metaanalyses of genome-wide association (GWA) studies are located at non-protein-coding regions. Here, we assessed the role of candidate regulatory variants associated with cell type-restricted, closely related hematological quantitative traits in biologically relevant hematopoietic cell types. We used formaldehyde-assisted isolation of regulatory elements followed by next-generation sequencing (FAIRE-seq) to map regions of open chromatin in three primary human blood cells of the myeloid lineage. In the precursors of platelets and erythrocytes, as well as in monocytes, we found that open chromatin signatures reflect the corresponding hematopoietic lineages of the studied cell types and associate with the cell typespecific gene expression patterns. Dependent on their signal strength, open chromatin regions showed correlation with promoter and enhancer histone marks, distance to the transcription start site, and ontology classes of nearby genes. Cell type-restricted regions of open chromatin were enriched in sequence variants associated with hematological indices. The majority (63.6\%) of such candidate functional variants at platelet quantitative trait loci (QTLs) coincided with binding sites of five transcription factors key in regulating megakaryopoiesis. We experimentally tested 13 candidate regulatory variants at 10 platelet QTLs and found that $10(76.9 \%)$ affected protein binding, suggesting that this is a frequent mechanism by which regulatory variants influence quantitative trait levels. Our findings demonstrate that combining large-scale GWA data with open chromatin profiles of relevant cell types can be a powerful means of dissecting the genetic architecture of closely related quantitative traits.
\end{abstract}

[Supplemental Material is available for this article.]

Genome-wide association (GWA) studies have discovered many non-protein-coding loci associated with complex traits. The precise localization of the causative sequence variant(s) at GWA loci is often impeded due to the extent of high linkage disequilibrium (LD), even when fine-mapping data are available. In addition, the functional impact of noncoding sequence variants at the molecular level is difficult to evaluate (Donnelly 2008; McCarthy et al.

\footnotetext{
${ }^{13}$ These authors contributed equally to this work.

${ }^{14}$ These authors jointly directed this work.

${ }^{15}$ A full list of members is provided in the Supplemental Material.

${ }^{16}$ Corresponding authors

E-mail d.paul@ucl.ac.uk

E-mail panos@sanger.ac.uk

Article published online before print. Article, supplemental material, and publication date are at http://www.genome.org/cgi/doi/10.1101/gr.155127.113. Freely available online through the Genome Research Open Access option.
}

2008; Cooper and Shendure 2011). Recent studies have shown that a large proportion of GWA signals are located within active gene regulatory elements in selected cell lines and primary tissues (The ENCODE Project Consortium 2012; Maurano et al. 2012). The ENCODE Project Consortium (2012) mapped deoxyribonuclease I (DNase I) hypersensitive and transcription factor binding sites in 147 cell types, and found that $34 \%$ and $12 \%$, respectively, of GWA lead SNPs overlapped with these regulatory regions. Maurano et al. (2012) expanded the catalog of DNase I hypersensitive sites to 349 cell types (including 85 ENCODE cell types), and showed that $57 \%$ of GWA lead SNPs were located within these regulatory sites. Additional candidate functional variants were retrieved by considering proxy SNPs that are in high LD with the lead SNP. Despite the ambitious scale of ENCODE and related efforts, biologically relevant effector (primary) cell types have not yet been assayed for many traits. 
We recently demonstrated that the formaldehyde-assisted isolation of regulatory elements (FAIRE) technique is a valuable tool in mapping nucleosome-depleted regions (NDRs) at selected genetic loci associated with hematological traits, and in prioritizing candidate variants for experimental validation (Paul et al. 2011). Hematological traits, such as the count and volume of cells in peripheral blood and the hemoglobin content of erythrocytes, are under genetic control and vary extensively between individuals (Evans et al. 1999; Garner et al. 2000). Such traits offer an excellent means of investigating the genetic architecture of closely related complex traits, because the cellular components of the hematopoietic system are well understood and primary precursor cells can be relatively easily accessed for experimental assays.

In this work, we used FAIRE-seq to map NDRs genome-wide in primary human megakaryocytes (MKs) and erythroblasts (EBs), the precursor cells of platelets and erythrocytes, respectively, as well as in monocytes (MOs). We also mapped NDRs in two immortalized cell lines commonly used as models for MKs and EBs, i.e., CHRF-288-11 and K562, respectively. First, we characterize the open chromatin profiles with respect to hematopoietic cell type and lineage, as well as FAIRE signal strength. Second, we assess the cell type-dependent enrichment patterns of sequence variants associated with two platelet and six erythrocyte indices at NDRs, using the results from the largest GWA meta-analyses conducted so far for these traits (Gieger et al. 2011; van der Harst et al. 2012). For these analyses, we also consider unrelated quantitative traits, i.e., fasting glucose (FG) and insulin (FI) levels, body mass index (BMI), and height (Dupuis et al. 2010; Lango Allen et al. 2010; Speliotes et al. 2010), as well as an open chromatin data set in a nonhematopoietic cell type, i.e., pancreatic islets (Gaulton et al. 2010). Finally, we experimentally validate a set of candidate regulatory variants identified within NDRs at platelet quantitative trait loci (QTLs).

\section{Results}

Preparation of open chromatin profiles of human myeloid cells

Cord blood-derived CD $34^{+}$hematopoietic progenitor cells (HPCs) from two unrelated individuals were differentiated in vitro into either MKs in the presence of thrombopoietin and interleukin-1 $\beta$, or into EBs in the presence of erythropoietin, interleukin-3, and KIT ligand (also known as stem cell factor). MOs were purified from peripheral blood from another two individuals (Supplemental Fig. 1A-C). In addition, we prepared FAIRE samples from CHRF-288-11 megakaryocytic cells and retrieved publicly available FAIRE-seq data for K562 erythroblastoid cells and pancreatic islets (Gaulton et al. 2010; The ENCODE Project Consortium 2012). Figure 1 gives an overview of the study design. All FAIRE-seq data sets were processed in a standardized manner, as described in the Methods section.

We determined FAIRE-derived NDRs (peaks) using a Gaussian kernel density estimator implemented in the software F-Seq (Supplemental Tables 1, 2; Boyle et al. 2008). As the Illumina DNA sequencing platform provides a large dynamic range and high sensitivity using discrete, digital sequencing read counts, we hypothesized that a subclassification of FAIRE peaks based on signal strength may allow more precise downstream functional analyses. Therefore, we stratified the peaks according to their signal strength (F-Seq peak score) into four equally spaced intensity bins, termed "Bins 1-4" (Supplemental Table 3).

The reproducibility of the peak calls across biological replicates in CHRF-288-11 and K562 cell lines increased with signal

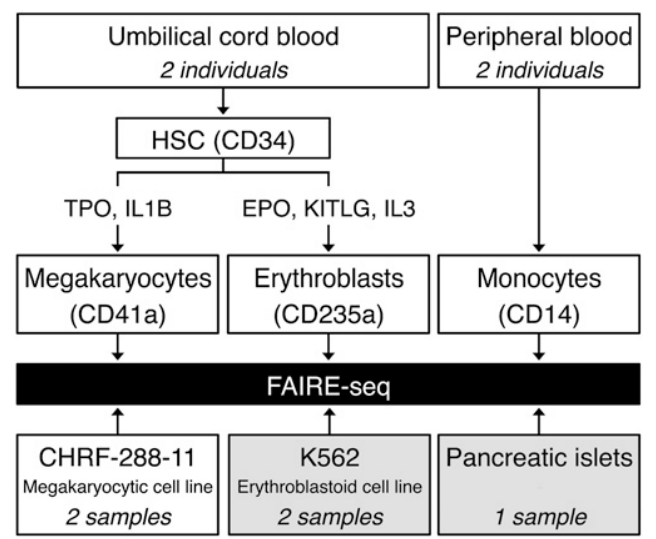

Figure 1. Overview of the study design. Cord blood-derived CD $34^{+}$ hematopoietic progenitor cells from two unrelated individuals were differentiated in vitro into either megakaryocytes (MKs) or erythroblasts (EBs). Monocytes (MOs) were purified from peripheral blood from another two individuals. We also prepared FAIRE samples from CHRF-288-11 megakaryocytic cells. In addition, we retrieved publicly available FAIREseq data sets for K562 erythroblastoid cells and pancreatic islets from The ENCODE Project Consortium (2012) and Gaulton et al. (2010), respectively, and reanalyzed the data sets in concordance with all other FAIRE data sets. (HSC) Hematopoietic stem cell; (TPO) thrombopoietin; (IL1B) interleukin 1, beta; (EPO) erythropoietin; (KITLG) KIT ligand (also known as SCF, or stem cell factor); (IL3) interleukin-3.

strength and was consistently $>80 \%$ for peaks in the top three intensity bins, i.e., Bins $2-4$. In contrast, we observed limited reproducibility $(<50 \%)$ of the peaks in the lowest intensity bin, i.e., Bin 1 (Supplemental Fig. 2A-K; Supplemental Table 4A). Furthermore, the fraction of overlap between peaks in Bin 1 and other regulatory marks ( $\mathrm{H} 3 \mathrm{~K} 4 \mathrm{me} 1 / 3$ histone modifications and transcription factor binding sites) was small compared with that of peaks in Bins 2-4 (Supplemental Table 4B); i.e., the overlap of FAIRE peaks with transcription factor binding sites in MKs was $4 \%$ in Bin 1 and on average $50 \%$ across Bins $2-4$. Thus, we excluded peaks in Bin 1 from subsequent analyses, as a large fraction of its peaks were neither reproducible nor appeared to bear hallmarks of regulatory chromatin.

\section{Hematopoietic cell type-restricted and lineage-restricted open chromatin signatures}

We first investigated to what extent individual myeloid cell types have distinct open chromatin signatures. We constructed distance matrices based on the overlap of NDRs across all sampled cell types (Supplemental Fig. 2A-K) and assessed the uncertainty of the clustering using bootstrap resampling (Suzuki and Shimodaira 2006).

We found that the hierarchical clustering is dominated by cell type identity rather than interindividual variation (Fig. 2A). The observed hierarchical tree branches reflected the established relation of the myeloid hematopoietic lineages. For example, MKs and EBs were found to cluster together, reflecting that the two cell types share a common progenitor, termed the MK-erythroid progenitor. MOs did not co-cluster with MKs/EBs and formed an outgroup, corresponding to the split of the common myeloid progenitor into the MK-erythroid and granulocyte-macrophage lineages. As expected, pancreatic islets formed another out-group (Supplemental Fig. 2K) due to the limited overlap between NDRs from endoderm-derived pancreatic islets and from mesoderm-derived hematopoietic cells. The difference in clustering between 
A

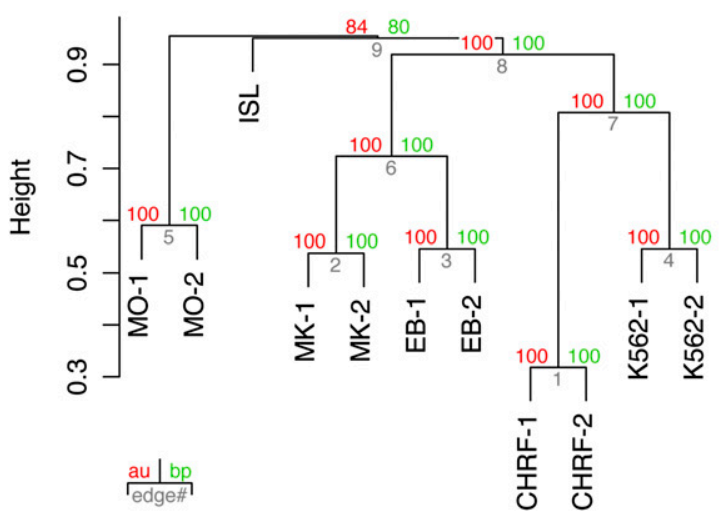

B

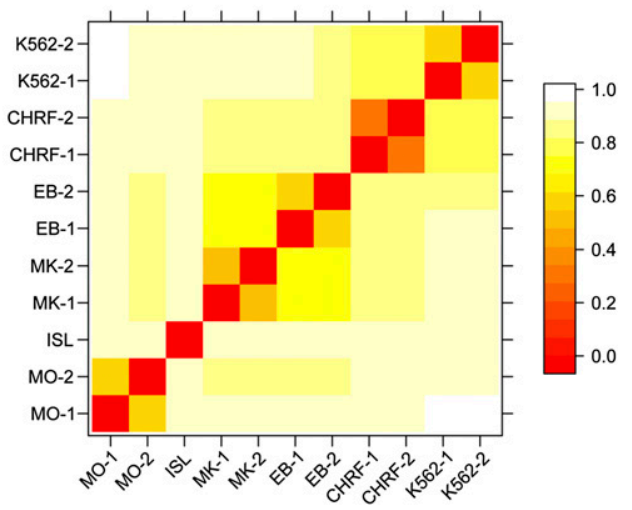

Figure 2. Hierarchical clustering of the overlap of FAIRE-derived nucleosome-depleted regions (NDRs). ( $A$ ) The hierarchical clustering is based on the overlap of NDRs across different cell types, as shown in Supplemental Figure 2. The dendrogram shows that the clustering is dominated by cell type identity rather than individual preparation. The observed hierarchical tree mirrors the hematopoietic tree, where MKs and EBs share a common progenitor. MKs and EBs do not co-cluster with their representative cell lines, i.e., CHRF-288-11 and K562, respectively, indicating that the open chromatin structure of immortalized lines does not fully reflect that of primary cells. Both MOs and pancreatic islets form out-groups, due to the limited overlap of NDRs with the other cell types tested. This suggests that MOs, despite being one of the myeloid types of cells akin to MKs and EBs, have a marked different open chromatin profile. The hierarchical cluster analysis was performed using the R package Pvclust (distance: binary; cluster method: complete) (Suzuki and Shimodaira 2006). The uncertainty of the clustering was assessed using bootstrap resampling. ( $B$ ) The heatmap of the binary distances complements the cluster plot. Relationships between NDRs across all samples are observable. The binary distances were plotted using the levelplot function of the R package lattice (http://cran.r-project.org/web/packages/lattice/). (MO) Monocyte; (MK) megakaryocyte; (EB) erythroblast; (ISL) pancreatic islet; (CHRF) CHRF228-11 megakaryocytic cell; (K562) K562 erythroblastoid cell; (au) approximately unbiased P-value; (bp) bootstrap probability value.

MOs and pancreatic islets as out-groups was marginal, based on their small number of shared NDRs across cell types (Supplemental Fig. 3). These data suggest that globally, the open chromatin signature of MOs is as unrelated to MKs/EBs as pancreatic islets, and indicate clear differences in chromatin profiles even within related hematopoietic cells of the same myeloid lineage.

We then compared the open chromatin profiles of MKs and EBs with that of CHRF-288-11 and K562 cells, respectively. The two investigated immortalized lines clustered closer to each other than to their respective primary cell type (CHRF-288-11/MKs and K562/ EBs), suggesting differences in open chromatin structure between immortalized lines and that of primary cells (Fig. 2A,B). For example, although there was extensive overlap between NDRs found in MKs and CHRF-288-11 cells (Supplemental Fig. 2A,B), the latter cell type possessed a large number of additional NDRs that overlapped with K562 cells but not with MKs (Supplemental Fig. 2G,H).

\section{Hematopoietic lineage-restricted gene expression patterns}

Next, we examined if the identified NDRs mark lineage-specific elements involved in regulation of expression of genes relevant to blood cell lineage commitment. For each cell type, we pooled the sequence fragments of the two individual FAIRE preparations and processed the data as for the individual preparations described above. We then assessed the expression levels of the single closest gene (defined as in terms of the distance to its transcription start site [TSS]) to each cell type-restricted NDR, interrogated over several time points during in vitro differentiation of HPCs into MKs (Supplemental Table 5A) and EBs (Supplemental Table 5B).

Transcripts close to MK-restricted NDRs (i.e., NDRs found only in MKs but not in the other cell types assayed) were more likely to be up-regulated during MK differentiation relative to all expressed transcripts (1.41-fold enrichment; $P=1.00 \times 10^{-29}$, twotailed $\chi 2$ test). We observed the same effect directionality for transcripts close to EB-restricted NDRs during EB differentiation $\left(1.50 ; P=1.23 \times 10^{-59}\right)$. Transcripts close to MO-restricted NDRs were down-regulated during both $\mathrm{MK}$ and $\mathrm{EB}$ differentiation with a fold change of $0.91\left(P=3.62 \times 10^{-9}\right)$ and $0.85\left(P=1.23 \times 10^{-19}\right)$, respectively.

Interestingly, transcripts in proximity to NDRs shared between MKs and MOs were also up-regulated during MK differentiation $\left(1.19 ; P=1.13 \times 10^{-5}\right)$. The corresponding genes $(n=265)$ mostly encode signaling proteins downstream from integrins, cytokine receptors, and $G$ protein-coupled receptors that are expressed in both MKs and MOs. We annotated the gene set using the Ingenuity Knowledge Base and found an enrichment of genes in the canonical pathways "Fc $\gamma$ receptor-mediated phagocytosis in macrophages and monocytes" $\left(P=4.53 \times 10^{-4}\right.$, BenjaminiHochberg corrected for multiple testing; $n=10$ genes) and "integrin signaling" $\left(P=4.53 \times 10^{-4}, n=14\right)$. We suggest that the 265 genes may also include a subset of coexpressed genes in MKs and MOs that play a role in the molecular events that link cell surface growth factor receptors to platelet integrin activation.

\section{Functional classification of NDRs based on signal strength}

We then investigated whether NDRs of different signal strength have different functional properties: in particular, their distance to the TSS, correlation with promoter and enhancer histone marks, and ontology classes of nearby genes.

First, we examined the location of NDRs of different signal strength relative to the TSS. We observed that for both MKs and EBs but not for MOs, NDRs in the lowest retained intensity bin (Bin 2; Supplemental Fig. 4A) were less often located close to the TSS than NDRs in the highest intensity bin (Bin 4; Supplemental Fig. 4B). We further investigated these observations by performing chromatin immunoprecipitation (ChIP) combined with high-throughput next-generation sequencing (ChIP-seq) of the histone modifications H3K4me3 and H3K4me1, which mark active promoters and enhancers, respectively. In MKs and EBs, NDRs in the highest in- 
tensity bin showed stronger overlap with promoters proximal to TSSs compared with NDRs at the other extreme, which showed stronger overlap with enhancers distal to TSSs (Fig. 3A,B). NDRs that did not overlap with either histone mark were more likely to be in the lowest intensity bin and located far from promoters. In contrast, we found that NDRs in the highest intensity bin in MOs were depleted close to the TSS compared with MKs and EBs (Fig. 3C).

Second, we applied the Genomic Regions Enrichment of Annotations Tool (GREAT) (McLean et al. 2010) to aid the functional interpretation of NDRs of different signal strength by analyzing the annotations of the single closest flanking gene (Supplemental Table 6). In MKs and EBs, NDRs in the lowest intensity bin were enriched in cell type-specific genes, while NDRs in the highest intensity bin were enriched in housekeeping genes. However, in MOs we observed an enrichment of cell type-specific gene sets close to NDRs irrespective of their signal strength. One possible explanation for the lack of enrichment in housekeeping genes could be that mature MOs, as studied here, do not proliferate but rather differentiate into various macrophage classes upon stimulation (Geissmann et al. 2010).

\section{Cell type-dependent enrichment of genome-wide significant SNPs associated with hematological traits at NDRs}

We assessed the enrichment of genome-wide significant SNPs associated with platelet and erythrocyte phenotypes at NDRs in a cell type-dependent context. We retrieved proxy SNPs $\left(r^{2}>\right.$ 0.8) (The 1000 Genomes Project Consortium 2010) of all GWA lead SNPs $\left(P<5 \times 10^{-8}\right)$ at 68 platelet and 75 erythrocyte QTLs (Gieger et al. 2011; van der Harst et al. 2012). By use of these criteria, we obtained 1680 and 4632 SNPs at platelet and erythrocyte QTLs, respectively. Then, we intersected the SNP positions with the composite map of open chromatin in myeloid cell types.

At $18(26.5 \%)$ and $25(33.3 \%)$ of the platelet and erythrocyte QTLs, respectively, we found at least one trait-associated SNP located within an NDR across MKs, EBs, and MOs (Fig. 4A,B; Supplemental Table 7A-D). Next, we compared the extent of overlap with 100,000 random sets of 68 and 75 SNPs that were matched for possible confounding factors such as minor allele frequency
(MAF), distance to a TSS, and number of proxy SNPs per locus (Methods). At platelet QTLs, significant overlap with NDRs in MKs $\left(P=2.0 \times 10^{-5}\right)$ and MOs $\left(P=1.7 \times 10^{-3}\right)$ was observed. The extent of overlap with NDRs in EBs was not more than expected by chance when compared to random sets of SNPs (Fig. 4C). At erythrocyte QTLs, we found significant $\left(P<1 \times 10^{-5}\right)$ overlap with NDRs in EBs, but not with NDRs in MKs or MOs (Fig. 4D). At both platelet and erythrocyte QTLs, there was no significant enrichment of GWA signals at NDRs in pancreatic islets.

Compared with immortalized cell lines representative of MKs and EBs, the same trends of enrichment as for the primary cell types were observed in platelet and erythrocyte traits, respectively. However, NDRs identified in CHRF-288-11 cells were also enriched for SNPs associated with erythrocyte indices (Fig. 4D). This is consistent with the notion that CHRF-288-11 cells are immature and therefore more closely related to MK-erythroid progenitor cells (Nürnberg et al. 2012), and is in agreement with the extensive overlap we found between NDRs in CHRF-288-11 and K562 cells (Supplemental Fig. 2G-J).

The NDRs overlapping platelet trait-associated SNPs were more likely to be restricted to MKs than expected by chance ( $P=$ $2.83 \times 10^{-4}$, Bonferroni-adjusted binomial test) (Fig. 5A; Supplemental Table 8A). We observed the same cell type-dependent effect for erythrocyte trait-associated SNPs at EB-restricted NDRs ( $P=$ $4.62 \times 10^{-7}$ ) (Fig. 5B; Supplemental Table 8B). These results suggest that regulatory variation may underlie the association signals observed at several of the genetic loci identified in hematological trait GWA studies. Importantly, the cell type corresponding to the hematological trait may play a pivotal role in the genetic architecture of the complex trait.

We further validated the statistical enrichment (Fig. 4C,D) by estimating the fold change of the number of GWA signals overlapping NDRs, relative to random sets of SNPs (Supplemental Fig. $5 \mathrm{~A}, \mathrm{~B})$. Despite the statistical enrichment and strong fold enrichment in relevant cell types, it is important to note that about half of the observed overlaps between candidate SNPs and NDRs are expected by chance (Supplemental Fig. 5A,B). Taken together, our findings suggest that the intersection of trait-associated SNPs with NDRs, and particularly for NDRs identified in relevant tissues, is likely to provide an informative ranking for selection of candidate causative variants for experimental validation.

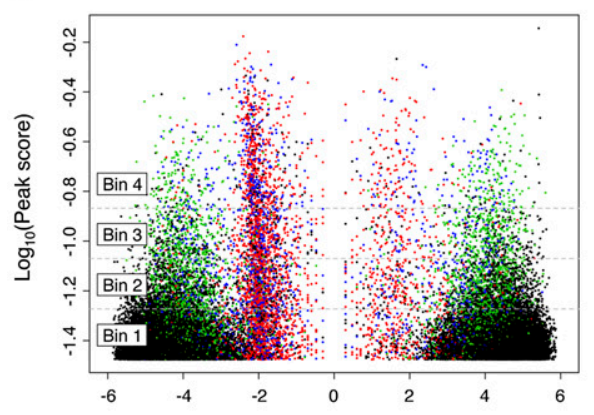

B

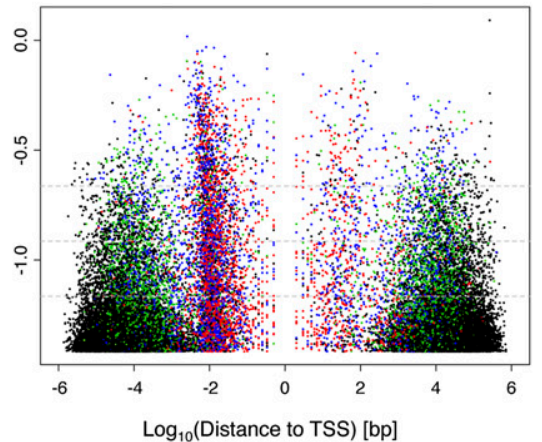

C

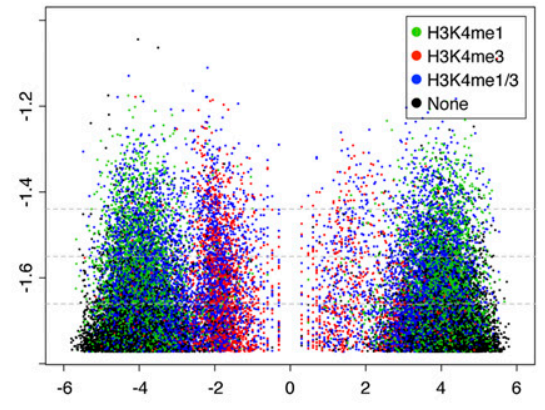

Figure 3. Overlap of $\mathrm{H} 3 \mathrm{~K} 4 \mathrm{me} 3$ (promoter) and $\mathrm{H} 3 \mathrm{~K} 4 \mathrm{me} 1$ (enhancer) histone marks with NDRs. In $(A)$ MKs and (B) EBs, NDRs in the highest intensity bin (Bin 4) showed stronger overlap with gene promoters close to TSSs compared with NDRs in the lowest retained intensity bin (Bin 2), which showed stronger overlap with enhancer elements distal to the closest TSS. NDRs that did not overlap with histone marks were more likely to be in the lowest intensity bin and far from promoters. (C) In MOs, however, we found that NDRs in the highest intensity bin were depleted close to the TSS compared with MKs and EBs. The peak bins are indicated with a dashed gray line. These results suggest that NDRs of different signal strength may have different functional properties. 
A

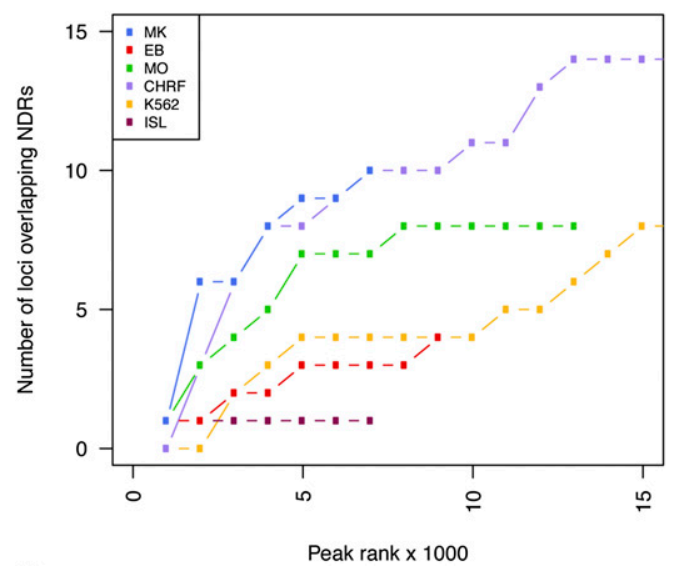

C

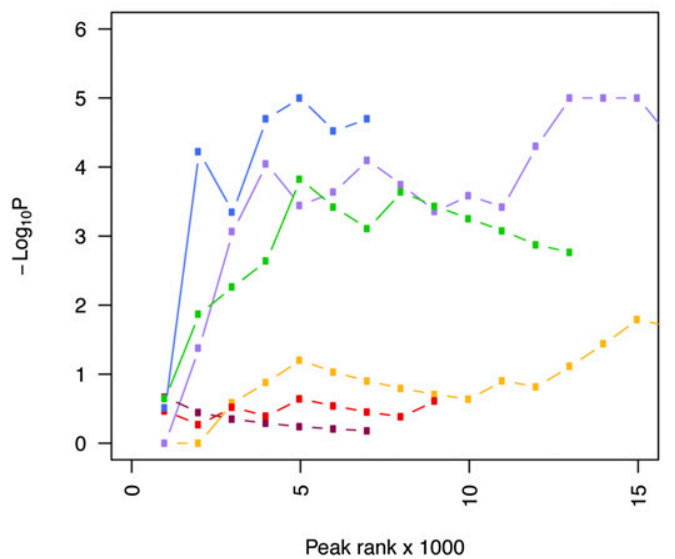

B

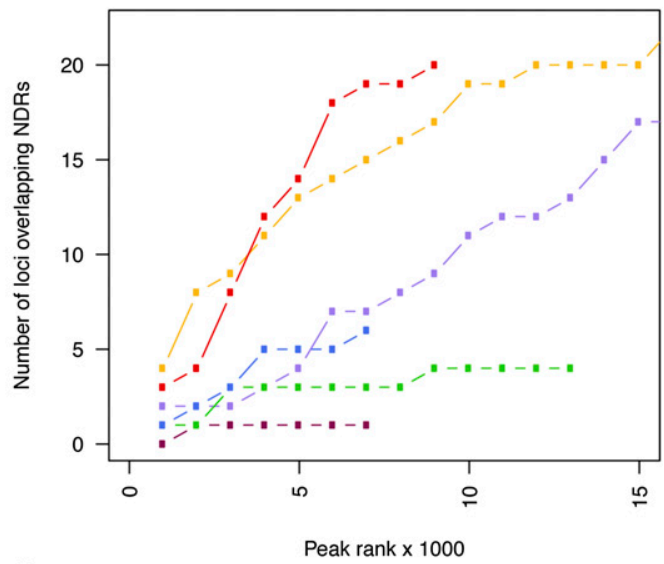

D

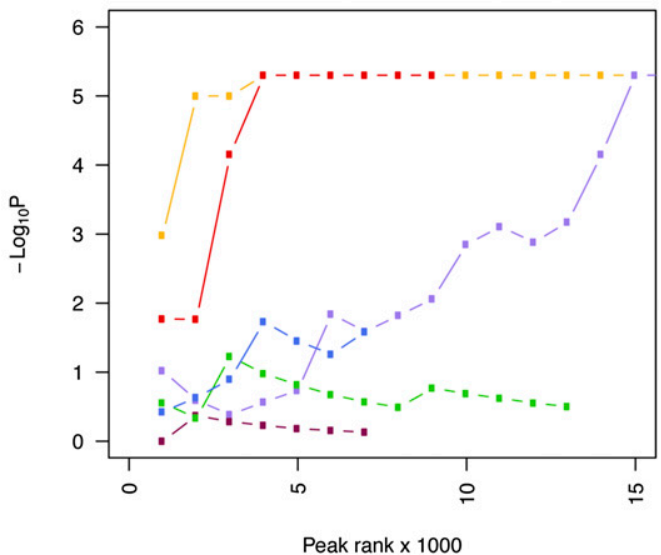

Figure 4. Cell type-dependent enrichment of GWA signals associated with hematological quantitative traits at NDRs. $(A, B)$ Cumulative number of GWA loci harboring platelet $(A)$ and erythrocyte $(B)$ trait-associated SNPs at NDRs across different cell types as a function of rank tranches for decreasing NDR signal strength (F-Seq peak score). (C,D) To determine whether such overlap was expected by chance, we compared the number of overlapping SNPs with 100,000 random samples of 68 and 75 SNPs at the platelet (C) and erythrocyte (D) QTLs, respectively. These random sets of SNPs were matched for possible confounding factors such as minor allele frequency, distance to a TSS, and number of proxy SNPs per locus. The achieved significance level is displayed across the cumulative rank tranches to better appreciate the effect of increasing the number of NDRs in the analysis. The strongest enrichment of genome-wide significant sequence variants at platelet and erythrocyte QTLs was found at NDRs in MKs and EBs, respectively. However, the enrichment was equally clear at NDRs in the respective immortalized lines, i.e., CHRF-288-11 megakaryocytic cells and K562 erythroblastoid cells, respectively. NDRs identified in CHRF-288-11 cells but not MKs were enriched for SNPs associated with erythrocyte indices, indicative of the less differentiated state of cell lines of leukemic origin relative to the primary cells.

\section{Identification of candidate functional variants at platelet QTLs}

To provide evidence that the SNPs we identified using the above approach are indeed valid functional candidates, we performed electrophoretic mobility shift assays (EMSAs). Here, we define "candidate functional variant" as a variant that affects DNA-protein binding, a possible mechanism for causality at non-protein-coding regions. We tested 13 functional candidates at 10 of the 18 identified platelet QTLS (Supplemental Table 9). These 13 selected SNPs were located within 11 NDRs that were present in both MKs and CHRF-288-11 megakaryocytic cells. Specifically, we identified two NDRs at the PTGES3-BAZ2A GWA locus, while the NDRs at the FAR2 and DNM3 loci each contained two candidate SNPs (Table 1). Importantly, seven of these 11 NDRs also coincided with binding sites of transcription factors key in regulating megakaryopoiesis (Tijssen et al. 2011), i.e., FLI1, GATA1, GATA2, RUNX1, and TAL1 (also known as SCL), suggesting identification of physiologically relevant regulatory elements.

For 10 of the 13 tested SNPs, we observed by visual inspection of the EMSA blot, differential binding of nuclear proteins between alleles in CHRF-288-11 cells (Supplemental Figs. 6A,B,E-I,N,O, 7B). For the three remaining SNPs, we observed comparable protein binding between allelic probes (Supplemental Fig. 6K-M).

We then annotated the candidate SNPs using RegulomeDB, a database containing known and predicted regulatory elements in the human genome (Boyle et al. 2012), and found all but one SNP to coincide with at least one RegulomeDB feature. Table 1 summarizes the obtained functional evidence for the platelet candidate variants.

As an example, the platelet count-associated SNP rs4148450 was located at an MK-restricted intronic NDR of ABCC4. The open chromatin region coincided with a RUNX1 transcription factor binding site in MKs (Supplemental Fig. 7A). ABCC4 encodes the ATP-binding cassette protein ABCC4, also known as multidrug resistance protein 4 (MRP4). Several studies indicated that ABCC4 is involved in the accumulation of the platelet-activating signaling molecule adenosine diphosphate (ADP) in platelet-dense granules (Jedlitschky et al. 2004, 2010). Our data suggest the noncoding SNP rs4148450 to be the functional variant at the $13 \mathrm{q} 32.1$ platelet

\section{Genome Research www.genome.org}


Table 1. Summary of the functional evidence obtained for platelet candidate functional SNPs through FAIRE, ChIP, and EMSA experiments, as well as annotation from RegulomeDB

\begin{tabular}{|c|c|c|c|c|c|c|c|}
\hline \multicolumn{3}{|c|}{ Candidate functional SNP } & \multirow[b]{2}{*}{ NDR cell type (Bin) } & \multirow{2}{*}{$\begin{array}{l}\text { GATA1/2, TAL1, RUNX1, } \\
\text { or FLI1 binding site in MKs }\end{array}$} & \multirow{2}{*}{$\begin{array}{l}\text { Binding } \\
\text { in } \mathrm{EMSA}^{\mathrm{b}}\end{array}$} & \multicolumn{2}{|r|}{ RegulomeDB annotation ${ }^{c}$} \\
\hline ID & Ref/alt & GWA locus & & & & Score & Supporting data \\
\hline rs1006409 & $\mathrm{A} / \mathrm{G}$ & FAR2 & MK (2) & - & Ref & $2 b$ & $\begin{array}{l}\text { TF binding }+ \text { any motif }+ \\
\text { DNase footprint }+ \text { DNase peak }\end{array}$ \\
\hline rs2015599a & $\mathrm{G} / \mathrm{A}$ & FAR2 & MK (2) & - & Ref & 4 & TF binding + DNase peak \\
\hline rs1107479 & $\mathrm{C} / \mathrm{T}$ & PTGES3-BAZ2A & MK (4)/EB (4) & - & Alt & $1 \mathrm{f}$ & eQTL + TF binding or DNase peak \\
\hline rs3214051 & $\mathrm{G} / \mathrm{A}$ & PTGES3-BAZ2A & MK (4)/EB (4)/MO (3) & FLI1 & Equal & 4 & TF binding + DNase peak \\
\hline rs17192586 & $\mathrm{G} / \mathrm{A}$ & RAD51B & MK (3) & RUNX1 & Alt & 4 & TF binding + DNase peak \\
\hline rs2038479a & $\mathrm{C} / \mathrm{A}$ & DNM3 & MK (3) & - & Ref & 5 & TF binding or DNase peak \\
\hline rs $2038480^{\mathrm{a}}$ & $A / T$ & DNM3 & MK (3) & - & Alt & 5 & TF binding or DNase peak \\
\hline rs214060 & $\mathrm{C} / \mathrm{T}$ & LRRC16A & MK (3) & - & Alt & 4 & TF binding + DNase peak \\
\hline rs3804749 & $\mathrm{C} / \mathrm{T}$ & PDIA5 & MK (4) & TAL1 & Equal & 5 & TF binding or DNase peak \\
\hline rs4148450 & $\mathrm{C} / \mathrm{T}$ & $A B C C 4$ & MK (2) & RUNX1 & Alt & 4 & TF binding + DNase peak \\
\hline rs55905547 & $\mathrm{A} / \mathrm{G}$ & CTSZ-TUBB1 & MK (3) & GATA1 + TAL1 & Equal & - & - \\
\hline rs6771416 & $\mathrm{G} / \mathrm{A}$ & KALRN & MK (2) & GATA1 + TAL1 & Alt & $2 b$ & $\begin{array}{l}\text { TF binding }+ \text { any motif }+ \\
\text { DNase footprint }+ \text { DNase peak }\end{array}$ \\
\hline rs7618405 & $\mathrm{C} / \mathrm{A}$ & SATB1 & MK (4) & GATA1 + RUNX1 + FLI1 + TAL1 & Ref & 4 & TF binding + DNase peak \\
\hline
\end{tabular}

(Ref) Reference allele. (Alt) Alternative allele. (TF) Transcription factor.

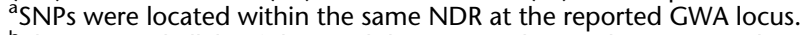

${ }^{\mathrm{b}}$ The reported allele of the candidate SNP indicates the EMSA probe with the stronger nuclear protein binding in CHRF-288-11 cells.

${ }^{\mathrm{C}}$ RegulomeDB score definition according to http://www.regulomedb.org/help\#score (Boyle et al. 2012).

count locus. As a further example, we recently described the molecular mechanism underlying the DNM3 platelet count and volume locus (Nürnberg et al. 2012). In brief, the SNP rs2038479 was located at intron 2 of DNM3, which encodes Dynamin 3, a mechanochemical enzyme involved in MK progenitor proliferation and maturation (Reems et al. 2008; Wang et al. 2011). The corresponding MK-restricted NDR (Table 1) was found to be bound by the MK-specific transcription factor MEIS1 and to mark an alternative promoter of a truncated DNM3 transcript, which is uniquely expressed in MKs and whose level depends on the rs2038479 genotype (Nürnberg et al. 2012).

Enrichment patterns of SNPs associated with closely related quantitative traits at cell type-restricted NDRs

Next, we investigated whether the various hematological parameters showed different patterns of enrichment at NDRs in the primary cells (see Fig. 6). In contrast to the analyses presented in Figure 4, where the different platelet and erythrocyte parameters were combined, here, we investigated the cellular parameters individually, and did not focus only on genome-wide significant signals. We considered sequence variants associated with two platelet indices (PLT, platelet count; MPV, mean platelet volume) and six erythrocyte indices $(\mathrm{Hb}$, total concentration of hemoglobin; PCV, packed red cell volume; RBC, red blood cell count; $\mathrm{MCHC}$, mean red cell hemoglobin concentration; $\mathrm{MCH}$, mean red cell hemoglobin; $\mathrm{MCV}$, mean red cell volume). In addition, we examined sequence variants associated with four nonhematological quantitative traits, i.e., FG and FI levels, BMI, as well as height (Dupuis et al. 2010; Lango Allen et al. 2010; Speliotes et al. 2010). As cell type-restricted NDRs showed stronger enrichment for sequence variants associated with the relevant trait compared with NDRs shared across cell types (Fig. 5A,B), we focused this in-depth analysis on NDRs restricted to MKs, EBs, MOs, or pancreatic islets.

To improve the statistical power of this analysis, we compared the distribution of $P$-values for all SNPs located at NDRs to the distribution of randomly selected SNP sets from the genome, matched for possible confounding factors (Methods). Specifically, we calculated the ratio at the 0.005 quantile between the $P$-value for a random, matched set of SNPs and the $P$-value for the SNPs within NDRs. Then, we estimated the ratio in 5000 bootstrap samples (Supplemental Fig. 8). In the absence of enrichment at NDRs, this ratio is expected to be one. Thus, this analysis quantifies to what extent SNPs at NDRs tend to have lower $P$-values than SNPs located outside NDRs, providing an indication of the extent to which SNPs at NDRs in a given cell type are enriched for potential causative variants compared with randomly selected SNPs. However, this analysis does not quantify the overall contribution from these variants to the phenotypic variation.

We observed diverse cell type-dependent enrichment patterns at NDRs for the various cellular parameters. For example, we found strong enrichment of SNPs associated with erythrocyte indices at EB-restricted NDRs. MCH and MCV, which are highly correlated quantitative traits $(r=0.91)$ (Supplemental Table 10), showed substantially stronger enrichment (ratios $>10^{6.2} \pm 1.5$ ) compared with the other four erythrocyte traits investigated (ratios $<10^{1.0 \pm 0.3}$ ). This suggests that MCH and MCV may be governed by molecular processes that are regulated at an intracellular level within the erythroid lineage. Conversely, variants associated with $\mathrm{Hb}$ were not significantly enriched in EB-restricted NDRs or any other cell type investigated. Indeed, hemoglobin concentration is tightly regulated by the level of bioavailable iron and is therefore dependent on several iron hemostasis processes involving the absorption, storage, and mobilization of iron. These processes comprise several organs in addition to erythroid cells, including the gut, the liver, and macrophages.

Both PLT and MPV association signals were enriched at MKrestricted NDRs. Interestingly, PLT-associated SNPs were also enriched at NDRs restricted to MOs and pancreatic islets. To shed light on the properties of the genes closest to these NDRs that contained a PLT-associated SNP $\left(P<10^{-4} ; n=75\right.$ genes), we performed canonical pathway analyses using the Ingenuity Knowledge Base. We detected a modest enrichment of genes involved in 
A

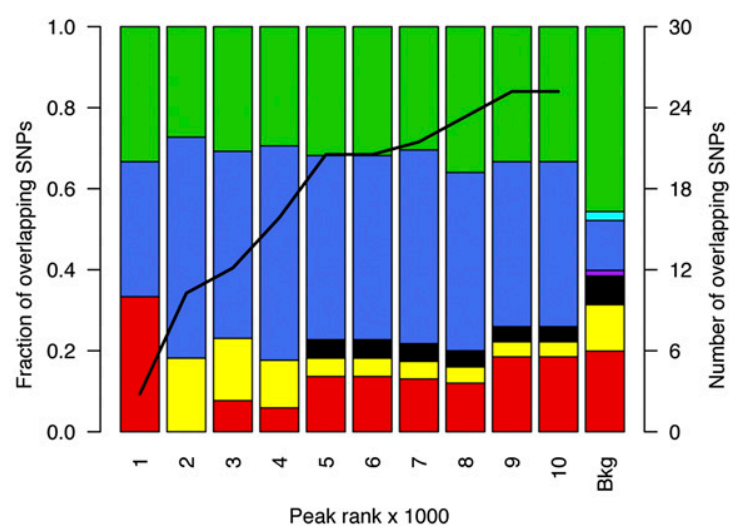

B

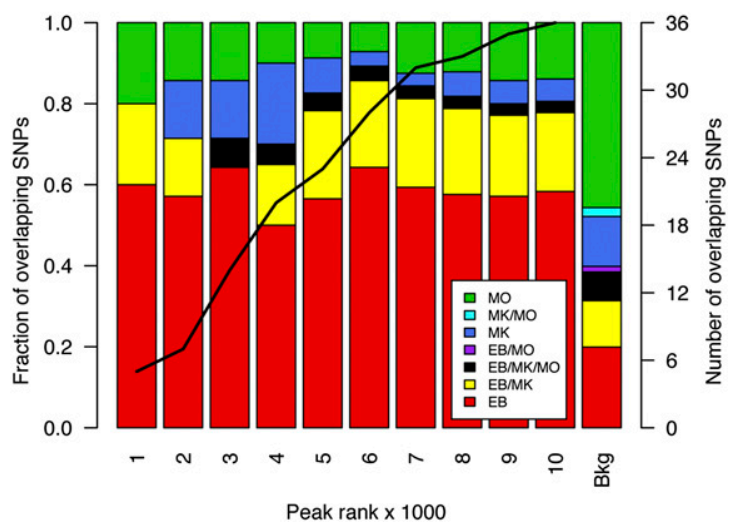

Figure 5. Cell type distribution of NDRs containing candidate functional variants. We considered GWA index SNPs associated with platelet $(A)$ and erythrocyte $(B)$ parameters, as well as their proxy SNPs in high $\operatorname{LD}\left(r^{2}>0.8\right.$; located within $1 \mathrm{Mb}$ of index SNPs). NDRs were ranked by signal strength (F-Seq peak score). Then, these rankings were used to divide the NDRs into cumulative tranches ( $x$-axis) to investigate the impact of peak calling thresholds on results. For example, the first bar represents the tranche containing the 1000 top-ranked NDRs, whereas the penultimate bar represents the tranche containing the 10,000 top-ranked NDRs of each cell type. The bars summarize the cell type distribution of candidate functional SNPs at NDRs as a percentage of the tranche-specific total. The last bar, labeled "Bkg," represents the expected cell type distribution for the SNPs under the null hypothesis. The solid line indicates the number of SNPs overlapping the tranche-specific NDRs. The results showed that for both platelet and erythrocyte QTLs, the candidate functional variants were most commonly found at MK- and EB-restricted NDRs, respectively. This was true across the spectrum of peak calling thresholds.

"cell-to-cell signaling and interaction" (range, $P=4.52 \times 10^{-2}$ to $1.17 \times 10^{-1}$, Benjamini-Hochberg corrected for multiple testing; $n=10$ ). Notably, these genes included THBS1 (encoding thrombospondin 1), WASL (Wiskott-Aldrich syndrome-like), and EDN1 (endothelin 1), which have a marked role in the activation of blood platelets (Dorahy et al. 1997; Falet et al. 2002; Jagroop et al. 2005). This suggests the involvement of non-cell-autonomous mechanisms for the regulation of platelet count, whereby extrinsic factors expressed by MOs or their progeny regulate the differentiation and proliferation of the hematopoietic stem cells toward MKs and the removal of senescent platelets from the circulation by liverresiding MO-derived macrophages.

The variation in enrichment patterns for cellular parameters of the same cell type was further illustrated by a trend of depletion of MCHC- and PCV-associated SNPs at MK-restricted NDRs. MCHand MCV-associated SNPs showed the opposite trend and were enriched at MK-restricted NDRs.

Some quantitative traits showed enrichment at NDRs in several different cell types. For example, PLT-associated SNPs were enriched at NDRs restricted to all four assayed cell types. A subset of platelet (i.e., PLT) and erythrocyte traits (i.e., $\mathrm{MCH}, \mathrm{MCV}, \mathrm{RBC}$, and PCV) was enriched at pancreatic islet-restricted NDRs.

SNPs associated with the four nonhematological quantitative traits tested were either not enriched or only weakly enriched at NDRs restricted to hematopoietic cells. Notably, height-associated SNPs were not significantly enriched at NDRs, even though this GWA study was very well powered. In contrast, FG-associated SNPs showed evidence for enrichment in three cell types. We note that the NDRs considered here may in fact be shared across other cell types not tested in this study, and thus, the corresponding gene expression pattern may be more global.

Taken together, these findings demonstrate that distinct cell type-restricted patterns can be identified for closely related quantitative traits, and for different hematological parameters of the same cell type, which we suggest reflect aspects of the different underlying molecular mechanisms. Thus, candidate variants may be subdivided based on their overlap with NDRs restricted to certain cell types, allowing for more informative downstream functional analyses.

\section{Discussion}

We generated genome-wide maps of open chromatin in human myeloid cells and used these to define cell type-dependent enrichment patterns of sequence variants associated with hematological quantitative traits at NDRs. These patterns allowed us to dissect platelet and erythrocyte quantitative trait associations in effector cell types within the myeloid arm of hematopoiesis.

Although immortalized cell lines are valuable tools for the discovery of NDRs, there were clear differences in patterns of chromatin accessibility compared with primary cells (Fig. 2A,B; Supplemental Table 2; Supplemental Fig. 2A-K). Among many factors, these differences may arise through serial subculturing of immortalized cell lines, resulting in a more homogenous cell population. Furthermore, the primary cells obtained by culture, i.e., MKs and EBs, will be more heterogeneous populations of cells at different stages of lineage commitment and maturation. Of the 68 and 75 GWA loci associated with platelet and erythrocyte traits, respectively, only five overlapped. This suggests that the effect of common variants on the formation of platelets and erythrocytes occurs after the MK-erythroid progenitor has committed to the megakaryocytic and erythroid lineages, making our findings with primary lineage-committed MKs and EBs particularly valuable for biological interpretation of NDRs.

We stratified NDRs based on their signal strength (peak score) into four intensity bins and excluded the lowest intensity bin (Bin 1) from further analyses due to the lack of reproducibility of FAIRE peaks between replicates, and limited overlap with other regulatory marks (Supplemental Table 4A,B). We recognize that division of peaks into four bins is arbitrary, but it represented the simplest approach to yield a sufficient number of peaks per bin to carry out the statistical analyses. With this caveat in mind, we provided 
evidence that NDRs of different signal strength have different functional features. For example, NDRs in the lowest retained intensity bin (Bin 2) were found to be located distal from the TSS and to overlap with H3K4me1 sites, similar to features of enhancer elements (Ernst and Kellis 2010; Ernst et al. 2011). Therefore, we suggest that stratification of peaks with respect to signal strength can be used to tailor downstream functional analyses.

We showed that there is cell type-dependent enrichment of hematological trait-associated variants at NDRs. For the genomewide significant SNPs, we found that $\sim 50 \%$ of the overlaps are not due to chance (estimated as the asymptotic enrichment of about two for unrelated cells in Supplemental Fig. 5A,B). Given our observation that $\sim 25 \%$ of the SNPs overlap a FAIRE peak (Fig. $4 A, B$ ), we suggest that $\sim 50 \% \times 25 \%=12.5 \%$ could be used as an estimate for the contribution from SNPs at NDRs to phenotypic variance explained by additive genetic effects using the cell types investigated. Since we did not find an overlap for every genome-wide significant SNP with a FAIRE peak, we do not claim that NDRs in the three primary cell types can explain all association signals.

We tested 13 candidate regulatory variants at platelet QTLs in EMSA studies, and provided evidence that all but three (76.9\%) of the tested SNPs exerted their effect through disruption or introduction of nuclear protein binding sites. This suggests that the impact of trait-associated sequence variants on protein binding sites may prove to be a key molecular mechanism at non-proteincoding regions. Indeed, results from our tightly focused analysis of hematopoietic QTLs confirm recent observations made by ENCODE and others that GWA variants frequently affect transcription factor occupancy as well as alter allelic chromatin states (The ENCODE Project Consortium 2012; Maurano et al. 2012). We tested by EMSA three additional candidate SNPs that were located within FAIRE peaks in the lowest intensity bin (Bin 1), which was excluded from analyses (see above). All three tested SNPs, i.e., rs11731274 and rs11734099 at the KIAA0232 gene locus and rs2735816 at the BRF1 locus (Supplemental Table 7A), did not show differential binding of nuclear proteins (Supplemental Fig. $6 \mathrm{C}, \mathrm{D}, \mathrm{J})$. Although these findings further justify our decision to exclude FAIRE peaks in Bin 1 from analyses, we also expect that a fraction of these peaks is likely to correspond to functional elements. A more refined approach, for example by incorporating overlaps with additional histone marks, will be needed to dissect more accurately this set of peaks.

As shown in Table 1, the functional evidence we obtained for the platelet candidate variants through EMSAs did not consistently correlate with publicly available regulatory annotation data sets. It is important to note that mere overlap of regulatory features (such as NDRs, transcription factor binding sites, and others) with a candidate SNP is not proof of a functional role of that SNP. For example, the SNP rs3214051 was located at an NDR shared across MKs, EBs, and MOs, and coincided with transcription factor binding sites, including FLI1, at the PTGES3-BAZ2A locus. However, EMSA experiments did not reveal differential protein binding between the allelic probes of the candidate SNP. Further experiments have to be carried out to investigate whether such SNPs affect the platelet phenotype through alternative molecular mechanisms. Several examples of such suitable experimental strategies have recently been described in the literature (Pomerantz et al. 2009; Tuupanen et al. 2009; Gaulton et al. 2010; Musunuru et al. 2010; Harismendy et al. 2011; Paul et al. 2011).

Sequence variants that are strongly associated with a quantitative trait but without necessarily surpassing the genome-wide threshold of significance $\left(P=5 \times 10^{-8}\right)$ are very likely to include additional true positive signals in well-powered GWA studies, such as the two large GWA meta-analyses we examined here (Gieger et al. 2011; van der Harst et al. 2012). There is an increasing body of evidence that functional data, e.g., gene expression QTLs, can be successfully correlated with GWA studies to reduce the false-discovery rate and identify novel association signals (Nicolae et al. 2010). In that context, we calculated the enrichment at the 0.005 quantile (Fig. 6; Supplemental Fig. 8), which places more emphasis on weaker regulatory associations that did not reach the threshold of genome-wide significance. This may explain the different results for the pancreatic islets, for which no enrichment was found using the genome-wide-associated SNPs (Fig. 4C,D; Supplemental Fig. 5A-D), while enrichment was found in the analysis that used all SNPs (Fig. 6). The observed enrichment of hematological traitassociated SNPs $\left(P>5 \times 10^{-8}\right)$ at cell type-restricted NDRs suggests that maps of open chromatin have the potential to pinpoint candidate sequence variants below the genome-wide significance threshold, effectively reducing the number of false-positive associations. Integration of such variants in network analyses and subsequent functional studies may provide valuable biological insights.

A more complete catalog of chromatin profiles will be needed to address whether the candidate functional SNPs have truly cell type-specific effects (i.e., out of all possible cell types). This can be addressed by large collaborative efforts such as ENCODE (The ENCODE Project Consortium 2012), BLUEPRINT (Adams et al. 2012), and the Roadmap Epigenomics Project (Bernstein et al. 2010). Incorporation of these genome- and epigenome-wide data sets in a multitude of different primary cell types will greatly facilitate the systematic functional interpretation of noncoding trait-associated sequence variants in terms of effector cell type and underlying molecular mechanism.

\section{Methods}

\section{$\mathrm{MO}$ isolation}

We isolated MOs from residual leukocytes obtained following apheresis platelet collections from Cambridge BioResource volunteers at NHS Blood and Transplant, Cambridge. Each sample (7.5 mL) was diluted 1:2 with PBE buffer (PBS [Sigma-Aldrich] at $\mathrm{pH}$ 7.2, 2 mM EDTA [Sigma-Aldrich], and 0.5\% BSA [Sigma-Aldrich]) and gently layered onto the membrane of a 50-mL Leucosep tube (Greiner Bio-One). Samples were centrifuged for $15 \mathrm{~min}$ at $800 \mathrm{~g}$ at room temperature (RT). The peripheral blood mononuclear cell (PBMC) layer was transferred into a fresh 50-mL tube. PBMCs from different Leucosep tubes were pooled, washed three times with $25 \mathrm{~mL}$ PBE buffer, and centrifuged for $5 \mathrm{~min}$ at $500 \mathrm{~g}$ at RT. PBMCs were counted, diluted to $1 \times 10^{8}$ cells $/ \mathrm{mL}$ with PBE buffer, and transferred into 5-mL polystyrene round-bottom tubes (BD Biosciences). MO isolation was performed using the EasySep Human CD14 Positive Selection Kit (StemCell Technologies) according to the manufacturer's instructions.

\section{MK and EB culture}

Umbilical cord blood was obtained after informed consent under a protocol approved by the NHS Cambridgeshire Research Ethics Committee (07/MRE05/44). Cord blood was collected into cord blood collection bags (MacoPharma). CD34 $4^{+}$HPCs were purified using the CD34 MicroBead Kit (Miltenyi Biotec) following the manufacturer's instructions. We tested purity (92\%-98\%) and viability of HPCs by flow cytometry. For in vitro differentiation of 


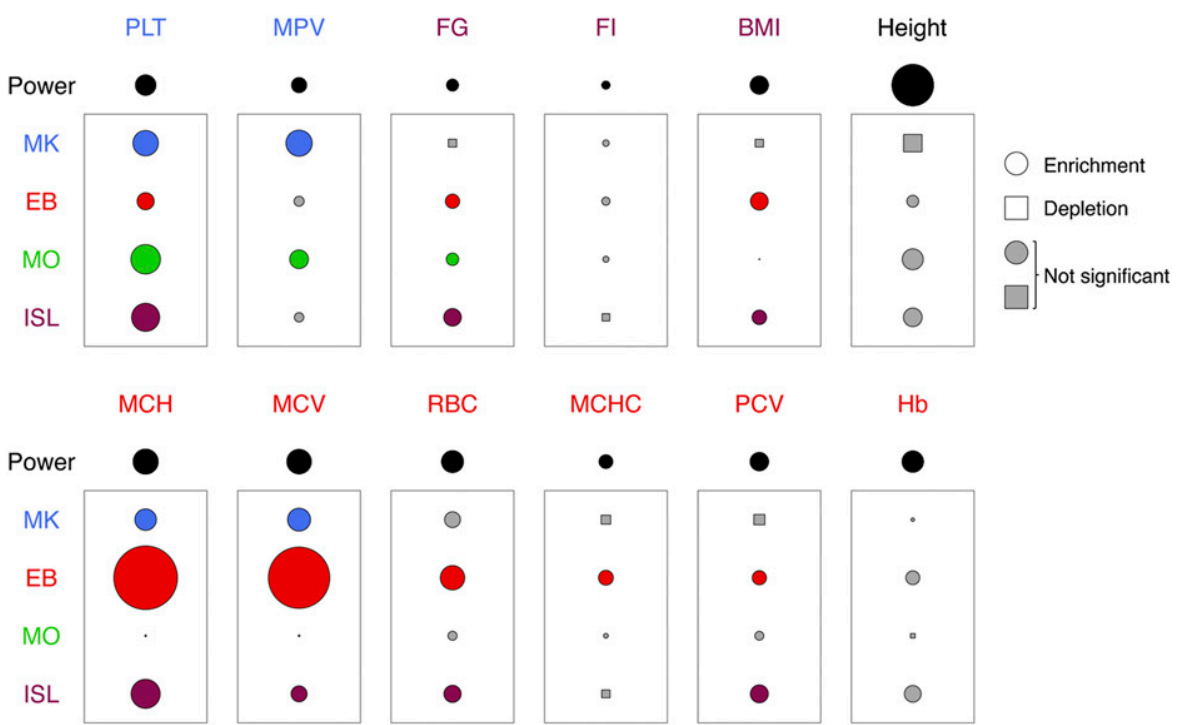

Figure 6. Enrichment patterns of quantitative trait-associated variants with small effect sizes at cell type-restricted NDRs. The data points shown as circles and rectangles represent the deviation of the $P$-value distribution of SNPs at NDRs restricted to MKs, EBs, MOs, or pancreatic islets (ISLs) from the $P$-value distribution of matched randomly sampled SNPs at the 0.005 quantile (Supplemental Fig. 8). Thus, this deviation measures the level of enrichment of associated sequence variants at NDRs, where the circle and rectangle surface areas represent level of enrichment (mean ratios $>1$ ) and depletion (mean ratios $<1$ ), respectively. Gray symbols represent ratios that are not significantly different from 1 ; i.e., the mean ratio across replicates was within 2 SDs of 1 . The level of enrichment is indicated for sequence variants associated with two platelet traits ([PLT] platelet count; [MPV] mean platelet volume), six erythrocyte indices $([\mathrm{Hb}]$ total hemoglobin concentration; $[\mathrm{PCV}]$ packed red cell volume; $[\mathrm{RBC}]$ red blood cell count; $[\mathrm{MCHC}]$ mean red cell hemoglobin concentration; $[\mathrm{MCH}]$ mean red cell hemoglobin; [MCV] mean red cell volume), as well as four nonhematological quantitative traits ([FG] fasting glucose; [FI] fasting insulin; [BMI] body mass index; height). The circle area labeled "Power" gives a quantification of the amount of signal present in each GWA data set. Specifically, it represents the deviation of the $P$-value distribution of all tested SNPs from the expectation under the null at the 0.005 quantile.

HPCs into MKs, 150,000 cells/mL/well were seeded in serum-free medium (CellGro SCGM, CellGenix) supplemented with $50 \mathrm{ng} / \mathrm{mL}$ human recombinant thrombopoietin (rhTPO; CellGenix) and $10 \mathrm{ng} / \mathrm{mL}$ interleukin 1, beta (rhIL-1 $\beta$; Miltenyi Biotech). To differentiate HPCs into EBs, we seeded 5000 cells $/ \mathrm{mL} /$ well in serum-free medium supplemented with 6 units $/ \mathrm{mL}$ erythropoietin (rhEPO; R\&D Systems), $10 \mathrm{ng} / \mathrm{mL}$ interleukin-3 (rhIL-3; Miltenyi Biotech), and $100 \mathrm{ng} / \mathrm{mL}$ stem cell factor (rhSCF; R\&D Systems). Cells were cultured for $10 \mathrm{~d}$ at $37^{\circ} \mathrm{C}$ and $5 \% \mathrm{CO}_{2}$. On the day of harvest, a cell aliquot was stained with $0.2 \%$ Trypan blue, and live cells were counted using a hemocytometer (InCyto C-Chip, VWR International).

\section{Cell morphology and flow cytometric analysis}

For cell morphological analysis, aliquots of 50,000 cells were centrifuged onto a glass slide for $5 \mathrm{~min}$ at $400 \mathrm{~g}$ at RT and stained with modified Wright's stain using an automated slide stainer (HemaTek 1000, Miles Laboratories). Stained cytospins were microscopically analyzed (Axiovert $40 \mathrm{CFL}$, AxioCam HSc, and AxioVision v4.5; Carl Zeiss MicroImaging). Aliquots of 300,000 cells were used for flow cytometry. We stained MOs with human anti-CD14-PE clone TUK4 and anti-CD45-FITC clone c29/33 (Alere), as well as FITC and PE mouse monoclonal IgG1 isotype control (BD Biosciences). After antibody incubation, $500 \mu \mathrm{L}$ washing buffer PBE buffer (PBS [Sigma-Aldrich] at pH 7.2, $2 \mathrm{mM}$ EDTA [Sigma-Aldrich], and 0.5\% BSA [Sigma-Aldrich]), and $5 \mu \mathrm{g} / \mathrm{mL}$ 7-amino actinomycin D (7-AAD; Invitrogen) were added. Flow cytometric analysis of MKs and EBs was performed according to the method previously described (Macaulay et al. 2007; Tijssen et al. 2011) using the following antibodies: human anti-CD41aAPC clone HIP8, anti-CD42a-FITC clone ALMA.16, anti-CD235a-FITC clone GA-R2 (HIR2), and anti-CD34-PE clone 581 (BD Biosciences). All samples were analyzed on the CyAn ADP 9-Color flow cytometer using the software Summit v4.3.02 (Beckman Coulter).

\section{Ploidy stain of MKs}

An aliquot of $1 \times 10^{6}$ MKs was fixed with $70 \%(w / v)$ ethanol (Sigma-Aldrich) for 30 min at RT, washed once with PBE buffer (PBS [Sigma-Aldrich] at $\mathrm{pH} 7.2,2 \mathrm{mM}$ EDTA [Sigma-Aldrich] and $0.5 \%$ BSA [Sigma-Aldrich]) and stained human antiCD41a-APC clone HIP8 (BD Biosciences) or matched isotype control, as described above. After centrifugation, cells were resuspended in $500 \mu \mathrm{L}$ staining buffer (465 $\mu \mathrm{L}$ PBE buffer, $5 \mu \mathrm{L}$ 10\% Tween-20 [Sigma-Aldrich], $5 \mu \mathrm{L}$ of $10 \mathrm{mg} / \mathrm{mL}$ RNase A [Sigma-Aldrich], and $25 \mu \mathrm{L}$ propidium iodide [Sigma-Aldrich]). After incubation for $30 \mathrm{~min}$ at $37^{\circ} \mathrm{C}$, DNA content was determined using a flow cytometer.

\section{Formaldehyde-assisted isolation of regulatory elements}

CHRF-288-11 cells were cultured according to the method previously described (Paul et al. 2011). We used approximately $10 \times 10^{6}$ CHRF-288-11 cells for each FAIRE experiment. Each FAIRE assay in primary human MKs, EBs, and MOs was performed with approximately $15 \times 10^{6}$ cells from two independent extractions. FAIRE was performed according to the method previously described (Paul et al. 2011), except that cross-linked washed cell pellets were resuspended in $2 \mathrm{~mL}$ of lysis buffer $(10 \mathrm{mM}$ Tris [Thermo Fisher Scientific] at $\mathrm{pH} 8.0,10 \mathrm{mM} \mathrm{NaCl}$ [VWR BDH Prolabo], $1 \times$ EDTA-free Protease Inhibitor [Complete Mini, Roche] and $0.2 \%$ Tergitol solution [Type NP-40, Sigma-Aldrich]). The sample was incubated for 10 min on ice. FAIRE DNA was processed following the Illumina paired-end library generation protocol. Genomic libraries derived from MO extractions and CHRF-288-11 cells were sequenced on Illumina HiSeq 2000 with 50-bp and 75-bp paired-end reads, respectively. Libraries derived from EB and MK cultures were sequenced on Illumina GAIIx with 54-bp paired-end reads.

\section{Sequence data processing}

Raw sequence reads were aligned to the human reference sequence (NCBI build 37) using the algorithm Stampy (Lunter and Goodson 2011). Reads were realigned around known insertions and deletions (The 1000 Genomes Project Consortium 2010), followed by base quality recalibration using the Genome Analysis Toolkit (GATK) (McKenna et al. 2010). Duplicates were flagged using the software Picard (http://picard.sourceforge.net/) and excluded from subsequent analyses. We retrieved FAIRE raw sequencing data for K562 erythroblastoid cells (GEO accession no. GSM864361) (The ENCODE Project Consortium 2012) and pancreatic islets (GEO

\section{Genome Research www.genome.org}


accession no. GSM491290) (Gaulton et al. 2010), and remapped the data as described above. An overview of the sequencing statistics is provided in Supplemental Table 1.

\section{Peak calling and binning strategy}

Regions of enrichment (peaks) were determined using the software F-Seq v1.84 (Boyle et al. 2008). We applied a feature length of $\mathrm{L}=$ $600 \mathrm{bp}$ and two different SD thresholds of $\mathrm{T}=6.0$ ("moderate") and $\mathrm{T}=8.0$ ("stringent") over the mean across a local background. In order to reduce false-positive peak calls, we removed regions of collapsed repeats according to the method recently described (Pickrell et al. 2011), applying a threshold of 0.1\% (http://eqtl. uchicago.edu/Masking). For comparison of open chromatin profiles, all read fragments were merged into one data set for each cell type. Then, peaks were called as described. For the K562 and pancreatic islets single-end sequencing data sets, we adjusted the mode of the peak width distribution to the mean of the modes across all non-K562 cells/pancreatic islets. We defined four equally spaced intensity bins between the first and 99th percentile of the $\log _{10}$-transformed F-Seq peak score distribution (termed "Bins $\left.1-4^{\prime \prime}\right)$. Then, we added the peaks below the first percentile and above the 99th percentile of the peak score distribution to Bin 1 and Bin 4, respectively. Supplemental Table 2 and Supplemental Table 3 give an overview of the peak data sets. ChIP-seq data sets in primary MKs were obtained from Tijssen et al. (2011) (GEO accession no. GSE24674). The peak coordinates were remapped to hg19 (minimum ratio of bases that must remap: 0.95) using the Lift-Over tool v1.0.3 of the web-based analysis platform Galaxy (http://main.g2.bx.psu.edu/).

\section{Hierarchical cluster analysis}

First, we created the union set of all peaks across all samples. Next, we defined a vector of binary values for each sample $s$, where the length of this vector is given by the total number of peaks in the union set and is therefore the same for all samples. Position $i$ in the vector for sample $s$ was set to a value of one, if the peak $i$ in the union peak set overlaps with a peak in sample $s$. If there was no overlap with a peak in sample $s$, position $i$ was set to zero. From these vectors, we constructed bin-specific vectors based on the binning scheme described above. For each bin and sample, we defined a vector where all entries with a peak score not between the lower and upper peak scores defined for that bin were set to zero. We then used the R package Pvclust (Suzuki and Shimodaira 2006) to perform a bootstrapped hierarchical cluster analysis of the samples based on these binary vectors, using the "binary" distance measure and the "complete" method for defining the clusters (Suzuki and Shimodaira 2006). Here, 1000 bootstrap samples were applied. All analyses were carried out in the R/Bioconductor environment.

\section{Gene expression analysis during in vitro differentiation of cord blood-derived HPCs}

Experiments and statistical analyses were performed according to the method previously described (Gieger et al. 2011). Briefly, MKs and EBs were differentiated from cord blood-derived HPCs as described above. Time points were taken at days 3, 5, 7, 9, 10, and 12 . Whole-genome gene expression levels were measured using Illumina HumanWG-6 v3 Expression BeadChips. Expressed probes were selected based on stringent thresholds, and the slope of expression was determined using standard linear regression. To every FAIRE peak, we assigned the single closest Ensembl transcript (release 69) with a HGNC symbol.

\section{H3K4mel and H3K4me3 ChIP}

MKs and EBs were differentiated from cord blood-derived HPCs as described above. ChIP assays were performed according to the method previously described (Forsberg et al. 2000), using rabbit polyclonal antibodies against H3K4me1 (ab8895, Abcam) and H3K4me3 (07-473, Millipore). Chromatin-immunoprecipitated DNA was sequenced on Illumina GAII with 54-bp single-end reads. Sequence reads were aligned using the algorithm BWA (Li and Durbin 2009). Areas of enrichment were determined using the slice function of the R package IRanges (http://bioconductor.org/packages/ 2.10/bioc/html/IRanges.html). Histone modification raw sequencing data for MOs were retrieved from ENCODE (GEO accession nos. GSM1003535 and GSM1003536) (The ENCODE Project Consortium 2012) and reanalyzed as described above. For H3K4me1, we identified 79,049 regions of enrichment in MKs, 66,410 in EBs, and 77,051 in MOs. For H3K4me3, 17,402 regions were found in MKs, 16,871 in EBs, and 33,842 in MOs.

\section{Annotation of NDRs using GREAT}

We analyzed the ontology of genes flanking FAIRE peaks using GREAT v1.8.2 (McLean et al. 2010) with the following parameters: association rule: single nearest gene; $1 \mathrm{Mb}$ maximal extension; curated regulatory domains excluded. The genomic distances between FAIRE peaks and TSSs were exported from the genomic region-gene association table in GREAT.

\section{Overlap of NDRs with genome-wide significant SNPs associated with hematological traits}

For each GWA locus, candidate functional SNPs were selected by identifying all biallelic SNPs with an $r^{2}>0.8$ and within $1 \mathrm{Mb}$ of the lead SNP in the European samples of the 1000 Genomes Project data set (interim phase I release of June 2011). We determined if at least one of these candidate SNPs overlapped with a FAIRE peak. Since this analysis is sensitive to the number of peaks, the overlap was carried out for a successively increasing number of peaks (cumulative tranches) by considering peaks with decreasing peak rank (F-Seq peak score). As more peaks are considered, the chance of finding an overlap increases. Therefore, we estimated the significance of our findings by resampling. Samples were drawn from the Phase II HapMap panel of approximately $2.6 \times 10^{6}$ SNPs (The International HapMap Consortium 2007), such that the MAF, the distance to a TSS, and the number of proxy SNPs $\left(r^{2}>0.8\right)$ had the same distribution as the genome-wide significant lead SNPs. This was achieved by estimating the joint distribution of the signed distance to a TSS and the number of proxy SNPs using a twodimensional Gaussian kernel density estimate as implemented in the R package KernSmooth (http://cran.r-project.org/web/packages/ KernSmooth/). The MAF was treated as an independent variable and was also estimated using a one-dimensional Gaussian kernel density estimate. We sampled with replacement 100,000 sets of lead SNPs (loci) of equal size as in the GWA study and with similar distribution of MAF, distance to TSS, and number of proxy SNPs per locus, representing loci drawn from the null distribution. Fold enrichment and $Z$-scores were calculated relative to the mean and $\mathrm{SD}$ of the observed number of GWA loci overlapping with an NDR for the null lead SNP sets. All analyses were carried out in the $\mathrm{R} /$ Bioconductor environment.

\section{Canonical pathway analysis using the Ingenuity Knowledge Base}

Genes were subjected to the core analysis module of the software Ingenuity IPA v14197757 (http://www.ingenuity.com) and analyzed 
using the following parameters: reference set: Ingenuity Knowledge Base (genes only); relationship to include: direct and indirect; filter: only molecules and/or relationships where species=human and confidence=experimentally observed. We report BenjaminiHochberg multiple test corrected $P$-values.

\section{Electrophoretic mobility shift assays (EMSAs)}

EMSAs using nuclear extracts from CHRF-288-11 cells were performed according to the method previously described (Paul et al. 2011). Oligonucleotide probes were designed based on the genomic sequence surrounding the candidate SNPs. A list of the oligonucleotides is provided in Supplemental Table 9. Competitor probes were prepared without biotin labels. All oligonucleotides were synthesized by Sigma-Aldrich. For competition assays, we used 100- or 200-fold molar excess (as indicated in Supplemental Figs. $6 \mathrm{~A}-\mathrm{O}, 7 \mathrm{~B}$ ) of the unlabeled probes.

\section{Enrichment analysis using bootstrapped quantile distributions}

The association analysis for the hematological quantitative traits was performed by imputation to the Phase II HapMap panel (The International HapMap Consortium 2007). To improve the coverage, we determined for each HapMap SNP which 1000 Genomes SNPs (interim phase I release of June 2011) within a distance of $50 \mathrm{~kb}$ had an $r^{2}>0.95$ with the imputed HapMap SNPs. For each trait, we assigned the $P$-value of the HapMap SNP from the metaanalysis to the 1000 Genomes SNP. To prevent chance inflation from LD and to obtain confidence estimates, SNPs were randomly removed until the genomic distance between remaining SNPs was at least $50 \mathrm{~kb}$. For each combination of trait and cell type, we created 5000 bootstrap samples of 1000 Genomes SNPs located at a cell type-restricted NDR. Then, for each bootstrap sample of SNPs located at an NDR, we created a matched null set of SNPs by sampling from the full set of 1000 Genomes SNPs that had an $r^{2}>$ 0.95 with a HapMap SNP. Each SNP was annotated for (1) MAF in the 1000 Genomes data set (bin boundaries: 0.0, 0.05, 0.1, 0.2, 0.3, $0.4,0.5,1.0)$; (2) genomic annotation (categories: exon, intron/ 3' UTR, promoter/TSS/5' UTR, intergenic/noncoding); (3) 36-bp mappability (bin boundaries $0.0,0.95,1.0$ ) (http://hgdownload. cse.ucsc.edu/goldenPath/hg19/encodeDCC/wgEncodeMapability/ wgEncodeCrgMapabilityAlign36mer.bigWig); and (4) absolute distance from the TSS of the nearest gene (bin boundaries: $0 \mathrm{~kb}$, $1 \mathrm{~kb}, 10 \mathrm{~kb}, 100 \mathrm{~kb}, 1 \mathrm{~Gb})$. We note that many of these categories are correlated, e.g., genomic annotation and distance to TSS. The distribution of annotation categories of SNPs in the null set was matched to that of the SNPs located at NDRs by sampling 10 random SNPs with the same annotation in the four categories for each SNP located at an NDR. The enrichment was quantified as the mean difference between the $-\log _{10}(P$-value $)$ at the 0.005 quantile in the sample of SNPs located at NDRs and the $-\log _{10}(P$ value) at the 0.005 quantile in the matched null set. The enrichment may be interpreted as the relative genomic inflation factor at the 0.005 quantile. The 0.005 quantile provides a trade-off between highlighting differences in enrichment between different cell types and reducing uncertainty in the estimates of the relative genomic inflation factors. All analyses were carried out in the R/Bioconductor environment.

\section{Data access}

The sequence data from this study have been submitted to the NCBI Gene Expression Omnibus (GEO) (http://www.ncbi.nlm. nih.gov/geo/) under accession no. GSE37916.

\section{Acknowledgments}

We thank S.F. Garner (Department of Haematology, University of Cambridge \& NHS Blood and Transplant, Cambridge, UK) and D.M. Bloxham (Department of Haematology, University of Cambridge \& Addenbrooke's Hospital, Cambridge) for support. We thank the core informatics, library-making, and sequencing teams at the Wellcome Trust Sanger Institute. D.S.P. and K.V. are supported by the Marie-Curie Initial Training Network NETSIM (EC-215820). D.S.P. is further supported by the EU-FP7 Project BLUEPRINT (282510). C.A.A. and A.R. are funded by the British Heart Foundation Program Grant RG/09/12/28096. J.S. and W.H.O. are supported by a grant from the National Institutes for Health Research (RP-PG-0310-1002). N.S. and P.D. are supported by the Wellcome Trust (098051).

Author contributions: D.S.P., K.V., and P.D. conceived and designed the experiments. D.S.P., K.V., and J.S. performed the experiments. C.A.A., A.R., and D.S.P. performed statistical analysis. C.A.A., A.R., D.S.P., and K.V. analyzed the data. P.vdH., J.C.C., and N.S. contributed GWA meta-analysis data sets. P.D. and W.H.O. jointly supervised the research. D.S.P. and P.D. prepared the manuscript, with major contribution from C.A.A., A.R., and W.H.O.

\section{References}

The 1000 Genomes Project Consortium. 2010. A map of human genome variation from population-scale sequencing. Nature 467: 1061-1073.

Adams D, Altucci L, Antonarakis SE, Ballesteros J, Beck S, Bird A, Bock C, Boehm B, Campo E, Caricasole A, et al. 2012. BLUEPRINT to decode the epigenetic signature written in blood. Nat Biotechnol 30: 224-226.

Bernstein BE, Stamatoyannopoulos JA, Costello JF, Ren B, Milosavljevic A, Meissner A, Kellis M, Marra MA, Beaudet AL, Ecker JR, et al. 2010. The NIH Roadmap Epigenomics Mapping Consortium. Nat Biotechnol 28: 1045-1048.

Boyle AP, Guinney J, Crawford GE, Furey TS. 2008. F-Seq: A feature density estimator for high-throughput sequence tags. Bioinformatics 24: 25372538 .

Boyle AP, Hong EL, Hariharan M, Cheng Y, Schaub MA, Kasowski M, Karczewski KJ, Park J, Hitz BC, Weng S, et al. 2012. Annotation of functional variation in personal genomes using RegulomeDB. Genome Res 22: 1790-1797.

Cooper GM, Shendure J. 2011. Needles in stacks of needles: Finding disease-causal variants in a wealth of genomic data. Nat Rev Genet 12: 628-640.

Donnelly P. 2008. Progress and challenges in genome-wide association studies in humans. Nature 456: 728-731.

Dorahy DJ, Thorne RF, Fecondo JV, Burns GF. 1997. Stimulation of platelet activation and aggregation by a carboxyl-terminal peptide from thrombospondin binding to the integrin-associated protein receptor. J Biol Chem 272: 1323-1330.

Dupuis J, Langenberg C, Prokopenko I, Saxena R, Soranzo N, Jackson AU, Wheeler E, Glazer NL, Bouatia-Naji N, Gloyn AL, et al. 2010. New genetic loci implicated in fasting glucose homeostasis and their impact on type 2 diabetes risk. Nat Genet 42: 105-116.

The ENCODE Project Consortium. 2012. An integrated encyclopedia of DNA elements in the human genome. Nature 489: 57-74.

Ernst J, Kellis M. 2010. Discovery and characterization of chromatin states for systematic annotation of the human genome. Nat Biotechnol 28: $817-825$.

Ernst J, Kheradpour P, Mikkelsen TS, Shoresh N, Ward LD, Epstein CB, Zhang X, Wang L, Issner R, Coyne M, et al. 2011. Mapping and analysis of chromatin state dynamics in nine human cell types. Nature 473: 43-49.

Evans DM, Frazer IH, Martin NG. 1999. Genetic and environmental causes of variation in basal levels of blood cells. Twin Res 2: 250-257.

Falet H, Hoffmeister KM, Neujahr R, Hartwig JH. 2002. Normal Arp2/3 complex activation in platelets lacking WASp. Blood 100: 2113-2122.

Forsberg EC, Downs KM, Bresnick EH. 2000. Direct interaction of NF-E2 with hypersensitive site 2 of the $\beta$-globin locus control region in living cells. Blood 96: 334-339. 
Garner C, Tatu T, Reittie JE, Littlewood T, Darley J, Cervino S, Farrall M, Kelly P, Spector TD, Thein SL. 2000. Genetic influences on F cells and other hematologic variables: A twin heritability study. Blood 95: 342-346.

Gaulton KJ, Nammo T, Pasquali L, Simon JM, Giresi PG, Fogarty MP, Panhuis TM, Mieczkowski P, Secchi A, Bosco D, et al. 2010. A map of open chromatin in human pancreatic islets. Nat Genet 42: 255-259.

Geissmann F, Manz MG, Jung S, Sieweke MH, Merad M, Ley K. 2010. Development of monocytes, macrophages, and dendritic cells. Science 327: 656-661.

Gieger C, Radhakrishnan A, Cvejic A, Tang W, Porcu E, Pistis G, SerbanovicCanic J, Elling U, Goodall AH, Labrune Y, et al. 2011. New gene functions in megakaryopoiesis and platelet formation. Nature 480: 201208.

Harismendy O, Notani D, Song X, Rahim NG, Tanasa B, Heintzman N, Ren B, Fu X-D, Topol EJ, Rosenfeld MG, et al. 2011. 9p21 DNA variants associated with coronary artery disease impair interferon- $\gamma$ signalling response. Nature 470: $264-268$.

The International HapMap Consortium. 2007. A second generation human haplotype map of over 3.1 million SNPs. Nature 449: 851-861.

Jagroop IA, Daskalopoulou SS, Mikhailidis DP. 2005. Endothelin-1 and human platelets. Curr Vasc Pharmacol 3: 393-399.

Jedlitschky G, Tirschmann K, Lubenow LE, Nieuwenhuis HK, Akkerman JWN, Greinacher A, Kroemer HK. 2004. The nucleotide transporter MRP4 (ABCC4) is highly expressed in human platelets and present in dense granules, indicating a role in mediator storage. Blood 104: 36033610.

Jedlitschky G, Cattaneo M, Lubenow LE, Rosskopf D, Lecchi A, Artoni A, Motta G, Nießen J, Kroemer HK, Greinacher A. 2010. Role of MRP4 (ABCC4) in platelet adenine nucleotide-storage. Am J Pathol 176: 10971103.

Lango Allen H, Estrada K, Lettre G, Berndt SI, Weedon MN, Rivadeneira F, Willer CJ, Jackson AU, Vedantam S, Raychaudhuri S, et al. 2010. Hundreds of variants clustered in genomic loci and biological pathways affect human height. Nature 467: 832-838.

Li H, Durbin R. 2009. Fast and accurate short read alignment with BurrowsWheeler transform. Bioinformatics 25: 1754-1760.

Lunter G, Goodson M. 2011. Stampy: A statistical algorithm for sensitive and fast mapping of Illumina sequence reads. Genome Res 21: 936939.

Macaulay IC, Tijssen MR, Thijssen-Timmer DC, Gusnanto A, Steward M, Burns P, Langford CF, Ellis PD, Dudbridge F, Zwaginga J-J, et al. 2007. Comparative gene expression profiling of in vitro differentiated megakaryocytes and erythroblasts identifies novel activatory and inhibitory platelet membrane proteins. Blood 109: 3260-3269.

Maurano MT, Humbert R, Rynes E, Thurman RE, Haugen E, Wang H, Reynolds AP, Sandstrom R, Qu H, Brody J, et al. 2012. Systematic localization of common disease-associated variation in regulatory DNA. Science 337: 1190-1195.

McCarthy MI, Abecasis GR, Cardon LR, Goldstein DB, Little J, Ioannidis JPA, Hirschhorn JN. 2008. Genome-wide association studies for complex traits: Consensus, uncertainty and challenges. Nat Rev Genet 9: 356-369.

McKenna A, Hanna M, Banks E, Sivachenko A, Cibulskis K, Kernytsky A, Garimella K, Altshuler D, Gabriel S, Daly M, et al. 2010. The Genome Analysis Toolkit: A MapReduce framework for analyzing nextgeneration DNA sequencing data. Genome Res 20: 1297-1303.
McLean CY, Bristor D, Hiller M, Clarke SL, Schaar BT, Lowe CB, Wenger AM, Bejerano G. 2010. GREAT improves functional interpretation of cisregulatory regions. Nat Biotechnol 28: 495-501.

Musunuru K, Strong A, Frank-Kamenetsky M, Lee NE, Ahfeldt T, Sachs KV, Li X, Li H, Kuperwasser N, Ruda VM, et al. 2010. From noncoding variant to phenotype via SORT1 at the 1p13 cholesterol locus. Nature 466: 714 719.

Nicolae DL, Gamazon E, Zhang W, Duan S, Dolan ME, Cox NJ. 2010. Traitassociated SNPs are more likely to be eQTLs: Annotation to enhance discovery from GWAS. PLoS Genet 6: e1000888.

Nürnberg ST, Rendon A, Smethurst PA, Paul DS, Voss K, Thon JN, LloydJones H, Sambrook JG, Tijssen MR, Italiano JE Jr, et al. 2012. A GWAS sequence variant for platelet volume marks an alternative DNM3 promoter in megakaryocytes near a MEIS1 binding site. Blood 120: 4859-4868.

Paul DS, Nisbet JP, Yang T-P, Meacham S, Rendon A, Hautaviita K, Tallila J, White J, Tijssen MR, Sivapalaratnam S, et al. 2011. Maps of open chromatin guide the functional follow-up of genome-wide association signals: Application to hematological traits. PLoS Genet 7: e1002139.

Pickrell JK, Gaffney DJ, Gilad Y, Pritchard JK. 2011. False positive peaks in ChIP-seq and other sequencing-based functional assays caused by unannotated high copy number regions. Bioinformatics 27: 21442146.

Pomerantz MM, Ahmadiyeh N, Jia L, Herman P, Verzi MP, Doddapaneni H, Beckwith CA, Chan JA, Hills A, Davis M, et al. 2009. The 8q24 cancer risk variant rs6983267 shows long-range interaction with MYC in colorectal cancer. Nat Genet 41: 882-884.

Reems J-A, Wang W, Tsubata K, Abdurrahman N, Sundell B, Tijssen MR, van der Schoot E, Summa FD, Patel-Hett S, Italiano JE Jr, et al. 2008. Dynamin 3 participates in the growth and development of megakaryocytes. Exp Hematol 36: 1714-1727.

Speliotes EK, Willer CJ, Berndt SI, Monda KL, Thorleifsson G, Jackson AU, Lango Allen H, Lindgren CM, Luan J, Magi R, et al. 2010. Association analyses of 249,796 individuals reveal 18 new loci associated with body mass index. Nat Genet 42: 937-948.

Suzuki R, Shimodaira H. 2006. Pvclust: An R package for assessing the uncertainty in hierarchical clustering. Bioinformatics 22: 1540-1542.

Tijssen MR, Cvejic A, Joshi A, Hannah RL, Ferreira R, Forrai A, Bellissimo DC, Oram SH, Smethurst PA, Wilson NK, et al. 2011. Genome-wide analysis of simultaneous GATA1/2, RUNX1, FLI1, and SCL binding in megakaryocytes identifies hematopoietic regulators. Dev Cell 20: 597-609.

Tuupanen S, Turunen M, Lehtonen R, Hallikas O, Vanharanta S, Kivioja T, Björklund M, Wei G, Yan J, Niittymäki I, et al. 2009. The common colorectal cancer predisposition SNP rs6983267 at chromosome 8q24 confers potential to enhanced Wnt signaling. Nat Genet 41: 885-890.

van der Harst P, Zhang W, Mateo Leach I, Rendon A, Verweij N, Sehmi J, Paul DS, Elling U, Allayee H, Li X, et al. 2012. Seventy-five genetic loci influencing the human red blood cell. Nature 492: 369-375.

Wang W, Gilligan DM, Sun S, Wu X, Reems J-A. 2011. Distinct functional effects for dynamin 3 during megakaryocytopoiesis. Stem Cells Dev 20: 2139-2151.

Received January 18, 2013; accepted in revised form April 2, 2013. 


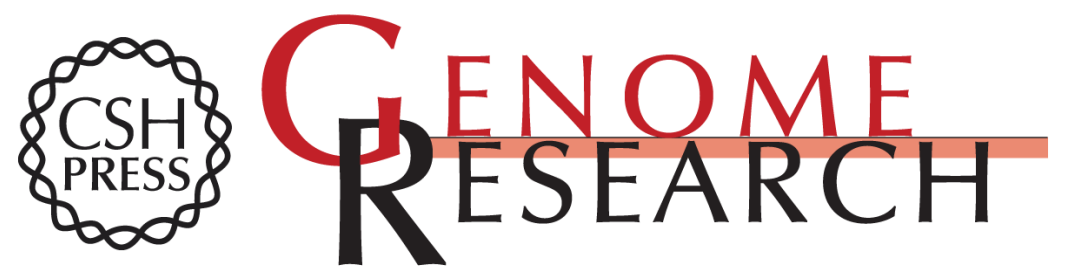

\section{Maps of open chromatin highlight cell type-restricted patterns of regulatory sequence variation at hematological trait loci}

Dirk S. Paul, Cornelis A. Albers, Augusto Rendon, et al.

Genome Res. 2013 23: 1130-1141 originally published online April 9, 2013

Access the most recent version at doi:10.1101/gr.155127.113

Supplemental Material

References

Open Access

Creative Commons

License

Email Alerting Service
http://genome.cshlp.org/content/suppl/2013/05/03/gr.155127.113.DC1

This article cites 45 articles, 12 of which can be accessed free at: http://genome.cshlp.org/content/23/7/1130.full.html\#ref-list-1

Freely available online through the Genome Research Open Access option.

This article, published in Genome Research, is available under a Creative Commons License (Attribution-NonCommercial 3.0 Unported), as described at http://creativecommons.org/licenses/by-nc/3.0/.

Receive free email alerts when new articles cite this article - sign up in the box at the top right corner of the article or click here.

\section{Affordable, Accurate Sequencing.}

